\title{
Potencial larvicida de Melia azedarach L. e Ilex paraguariensis A. St.-Hil. no controle de Aedes aegypti (Linnaeus, 1762) (Diptera: Culicidae)
}

\author{
Larvicidal potential of Melia azedarach L. and Ilex paraguariensis A. St.-Hil. in the \\ control of Aedes aegypti (Linnaeus, 1762) (Diptera: Culicidae). \\ Maria Assunta Busato*1, Jaqueline Vitorello², Junir Antonio Lutinski ${ }^{3}$, \\ Jacir Dal Magro ${ }^{4}$ e Jaqueline Scapinello ${ }^{5}$ \\ ${ }^{1}$ Doutora em Biologia. Docente, Universidade Comunitária da Região de Chapecó, SC,Brasil \\ ${ }^{2}$ Graduanda em Ciências Biológicas na Universidade Comunitária da Região de Chapecó, SC,Brasil \\ ${ }^{3}$ Doutor em Biodiversidade Animal. Docente, Universidade Comunitária da Região de Chapecó, SC,Brasil \\ ${ }^{4}$ Doutor em Química. Docente, Universidade Comunitária da Região de Chapecó, SC,Brasil \\ ${ }^{5}$ Mestre em Ciências Ambientais. Docente Universidade Comunitária da Região de Chapecó, SC,Brasil
}

\begin{abstract}
Resumo
O mosquito Aedes aegypti é o principal vetor da dengue. Os produtos sintéticos convencionais utilizados no controle populacional deste vetor têm apresentado resistência das populações, além de riscos à saúde da população e dos próprios aplicadores. Esse estudo se propôs a avaliar o potencial larvicida de extratos etanólicos de Ilex paraguariensis e Melia azedarach no controle de A. aegypti em condições de laboratório. Os ensaios foram conduzidos em recipientes plásticos com capacidade de $150 \mathrm{ml}$, onde foram colocados $60 \mathrm{ml}$ das soluções contendo concentrações diferentes dos extratos. A esta solução foram adicionadas 10 larvas ativas dos instares L2 e L3. O experimento foi realizado em triplicata, utilizado um controle contendo apenas água e alimento. As avaliações de susceptibilidade foram realizadas com 24 h e $48 \mathrm{~h}$ após a aplicação. Os tratamentos foram comparados entre si com base no número de larvas vivas. A eficiência dos tratamentos testados foi registrada por meio do percentual de mortalidade calculado pela equação de Abbott (1925). O potencial larvicida dos tratamentos foi de 100\% para a concentração de $500 \mu \mathrm{g} / \mathrm{mL}$ para o extrato dos frutos de M. azedarach (48 h) e $2000 \mu \mathrm{g} / \mathrm{mL}$ para os extratos das folhas de I. paraguariensis (24 h) e M. azedarach (48 h). A eficiência dos extratos de I. paraguariensis e M. azedarach são resultados promissores. A possibilidade do uso dos extratos destas plantas como larvicida para A. aegypti representa uma alternativa frente ao produto sintético recomendado pelo Ministério da Saúde para o controle da dengue.
\end{abstract}

Palavras-chave: Vetor. Produtos naturais. Extratos. Erva-mate

\begin{abstract}
The mosquito Aedes aegypti is the main vector of dengue. The conventional synthetic products used in the population control of this vector have presented resistance of the populations, in addition to risks to the health of the population and of the applicators themselves. This study was aimed at assessing the larvicidal potential of ethanolic extracts of Ilex paraguariensis and Melia azedarach in the control of A. aegypti in laboratory conditions. The assays were performed in plastic containers with a capacity of $150 \mathrm{ml}$, where were put $60 \mathrm{ml}$ of the solutions containing different concentrations of the extracts. To this solution, 10 active larvae of the instars L2 and L3 were added. The experiment was accomplished in triplicate by using a control containing only water and food. The susceptibility assessments were accomplished at $24 \mathrm{~h}$ and $48 \mathrm{~h}$ after application. The treatments were compared with basis on the amount of live larvae. The efficiency of the tested treatments was recorded by means of the percentage of mortality calculated by the Abbott's formula (1925). The larvicidal potential of the treatments was $100 \%$ for the concentration of $500 \mu \mathrm{g} / \mathrm{mL}$ for the extract of the fruits of M. azedarach (48 h) and $2000 \mu \mathrm{g} / \mathrm{mL}$ for the extracts of the leaves of $\mathrm{I}$. paraguariensis $(24 \mathrm{~h})$ and $\mathrm{M}$. azedarach $(48 \mathrm{~h})$. The efficiency of the extracts of $\mathrm{I}$. paraguariensis and $\mathrm{M}$. azedarach are promising results. The possibility of use of the extracts of these plants as larvicidal agents for A. aegypti is an accessible alternative before the synthetic product recommended by the Ministry of Health to control dengue.
\end{abstract}

Keywords: Vector. Natural products. Extracts. Mate herb 


\section{Introdução}

$\mathrm{O}$ controle natural de vetores com o uso de produtos naturais é menos impactante em relação aos inseticidas convencionais (sintéticos), pois esses produtos apresentam uma degradação mais rápida, resultando em menor exposição ocupacional e poluição do meio ambiente (BARRETO, 2005). A utilização de produtos de origem vegetal para o controle de pragas tem sido amplamente estudada devido ao potencial dessas substâncias como alternativas aos compostos sintéticos disponíveis no mercado, à elevada taxa de biodegradabilidade no ambiente e à menor toxicidade sobre espécies não alvo (VALLADARES et al., 1997; BEZERRA et al., 2014; KRINSKI, MASSAROLI, MACHADO, 2014). Vários pesquisadores têm trabalhado com produtos naturais, visando obter extratos de plantas, com atuação no desenvolvimento embrionário, na fase larval, pupa e na emergência dos adultos de insetos, inclusive o A. aegypti (POHLIT et al., 2004; BRUNHEROTTO e VENDARAMIM, 2001; CORIA et al., 2008; VALLADARES et al., 1997).

O mosquito $A$. aegypti é uma das mais de 500 espécies do gênero Aedes (Diptera: Culicidae). É o principal vetor da dengue e utiliza depósitos de água para colocar os seus ovos. Estes possuem capacidade de resistir à dessecação, mantendo-se viáveis na ausência de água por mais de um ano (TAUIL, 2002). Quando os ovos entram em contato com a água, após dois a quatro dias dão origem às larvas, que passam por quatro estágios: L1, L2, L3 e L4. Posteriormente, originam as pupas e, logo após, o mosquito adulto (NEVES, 2005). O controle populacional tem se concentrado sobre as fases larvais quando o processo é mais eficiente, mais seletivo e menos oneroso aos programas de prevenção à dengue (SANTA CATARINA, 2014).

Dentre as estratégias para controle populacional do vetor da dengue, ainda ocorre a utilização em larga escala de inseticidas sintéticos, como é o caso do organofosforado temefós. O uso deste inseticida pode acarretar problemas de intoxicação e contaminação ambiental, o que ocasiona riscos à biodiversidade local, bem como à saúde humana (LASNEAUX, 2013; AUGUSTO; CÂMARA NETO, 2005). Para a saúde ambiental, o uso contínuo desse inseticida pode apresentar efeitos indesejáveis, como a permanência por longos períodos de tempo no ambiente, a seleção de populações de vetores resistentes e o aparecimento de novas pragas (DONALÍSIO; GLASSER, 2002). Além da resistência associada à utilização indiscriminada de produtos sintéticos no controle de vetores, pode ocorrer a eliminação de espécies que contribuem para o equilíbrio ambiental (BARRETO, 2005).

Conhecer o efeito larvicida de produtos naturais pode ser uma alternativa viável, pois tais produtos podem causar menos impactos quando comparados aos larvicidas convencionais. Nesta perspectiva, este estudo se propôs a avaliar o potencial larvicida de extratos vegetais de $M$. azedarach (conhecida popularmente como cinamomo) e de I. paraguariensis (erva-mate) sobre A. aegypti.

\section{Materiais e métodos}

A coleta dos ovos de A. aegypti foi realizada no período de novembro de 2013 a março de 2014. Foram instaladas ovitrampas em diferentes pontos da cidade de Chapecó - SC, contemplando as regiões da cidade com maior índice de infestação pelo mosquito, segundo os dados do Programa de Controle da Dengue (SANTA CATARINA, 2014). A ovitrampa consiste em um pote de plástico preto, com capacidade de $300 \mathrm{ml}$. Em cada ovitrampa foram acondicionadas quatro palhetas de Eucatex e $150 \mathrm{ml}$ de uma solução atrativa (obtida a partir de feno fermentado). As ovitrampas permaneceram no campo de 3 a 5 dias. As palhetas recolhidas foram identificadas, com a localidade de procedência e a data, e secas em laboratório, à temperatura ambiente, por 48 horas. Os ovos foram contados com utilização de lupa e armazenados em sacos plásticos.

Para obtenção dos extratos, foram utilizados frutos e folhas de M. azedarach e I. paraguariensis, coletados em fevereiro e junho de 2013, respectivamente, e folhas de I. paraguariensis, que foram coletadas no mês de agosto de 2013, ambas no município de Chapecó, SC (27ํㅜ 05' $35,23^{\prime \prime}$ 'S e 52 39' 52" O).

Após a coleta, o material vegetal foi seco em estufa a $40{ }^{\circ} \mathrm{C}$, durante $24 \mathrm{~h}$, e triturado. Os extratos dos frutos e folhas de $M$. azedarach foram obtidos por maceração em álcool etílico PA, na proporção de 1:3 gramas de material vegetal por litro de solvente, durante 48 horas, e homogeneizados em intervalos de 12 horas. Após este período, o macerado foi filtrado para posterior concentração em evaporador rotatório, sob pressão reduzida, e, então, liofilizado, originando o extrato livre de solvente utilizado nos testes.

As concentrações de extrato avaliadas foram 100, 250, 500,1000 e $2000 \mu \mathrm{g} / \mathrm{ml}$, para as folhas de M. azedarach e I. paraguariensis, e 100, 250 e $500 \mu \mathrm{g} / \mathrm{ml}$, para os frutos de M. azedarach. Para a concentração de $100 \mu \mathrm{g} / \mathrm{ml}, 0,0250 \mathrm{~g}$ de extrato foram previamente solubilizados em Tween-20 e, em seguida, adicionados $250 \mathrm{ml}$ de água ultrapura e homogeneizado. O mesmo procedimento foi realizado para as demais concentrações, variando a quantidade de extrato conforme a concentração preparada em 250 $\mathrm{ml}, 0,125 \mathrm{~g}$ para $500 \mu \mathrm{g} / \mathrm{ml} ; 0,250 \mu \mathrm{g} / \mathrm{ml}$ para $1000 \mu \mathrm{g} / \mathrm{ml}$ e $0,500 \mathrm{~g}$ para $2000 \mu \mathrm{g} / \mathrm{ml}$.

Os bioensaios foram realizados em ambiente de laboratório, sob condições controladas de temperatura $\left(27^{\circ} \mathrm{C}\right.$ a $30^{\circ} \mathrm{C}$ ) e fotoperíodo ( 12 horas) até a eclosão dos ovos. Para obtenção das larvas, as palhetas com o número de ovos determinados foram colocadas em bandejas com água sem tratamento. As larvas foram alimentadas com 
ração de peixe e permaneceram nas bandejas até atingir os instar L2 e L3, cujo período médio foi de quatro dias.

Os ensaios foram conduzidos em recipientes plásticos, com capacidade de $150 \mathrm{ml}$. Nestes, foi adicionado $60 \mathrm{ml}$ de solução nas diferentes concentrações dos extratos e 10 larvas ativas dos instares L2 e L3. A testemunha conteve apenas água e alimento. $\mathrm{O}$ experimento foi realizado em triplicata. A susceptibilidade das larvas foi avaliada após $24 \mathrm{~h}$ e $48 \mathrm{~h}$ de exposição às soluções. A mortalidade foi comprovada quando as larvas não apresentaram movimentos a estímulos mecânicos.

A avaliação dos dados foi feita a partir da análise de variância sobre o número de larvas vivas, sendo que as médias foram agrupadas pelo teste de Duncan em nível de $5 \%$ de probabilidade de erro. Os valores foram previamente transformados em $\log (x+1)$. A eficiência dos tratamentos testados foi calculada pela equação de Abbott (1925).

\section{Resultados}

As larvas de A. aegypti demonstraram suscetibilidade aos extratos e concentrações testadas. Também foi observada variação na mortalidade larval nos períodos de exposição avaliados (24 h e 48 h) (Tabela 1).

Para o extrato dos frutos de $M$. azedarach, foi possível observar que este causou a morte das larvas a partir da concentração de $100 \mu \mathrm{g} / \mathrm{ml}$. A susceptibilidade de 100\% foi registrada na concentração de $500 \mu \mathrm{g} / \mathrm{ml}$. As folhas da M. azedarach também apresentaram eficiência larvicida, com ação nas primeiras $24 \mathrm{~h}$ a partir da concentração de $100 \mu \mathrm{g} / \mathrm{ml}$, alcançando $100 \%$ de eficiência no tratamento com $2000 \mu \mathrm{g} / \mathrm{ml}$ (Tabela 1).

No período de exposição dos extratos por $24 \mathrm{~h}$, foram identificadas oscilações na mortalidade das larvas, a partir das diferentes concentrações, conforme observado na Tabela 1. Contudo, após 48 horas da aplicação, a susceptibilidade aumentou conforme a maior concentração dos extratos (Figura 1).

A mortalidade larval com extrato de folhas e de $I$. paraguariensis ocorreu a partir da concentração de 250 $\mu \mathrm{g} / \mathrm{ml}$, e a letalidade de $100 \%$ em 2000 g/ml (Tabela 1). Em 48h de exposição, os extratos apresentaram 100\% de eficiência, mesmo nas concentrações mais baixas, para os extratos das duas plantas utilizadas.

Tabela 1 - Eficiência larvicida de extratos de M. azedarach e I. paraguariensis em diferentes períodos de exposição, 24h e 48h. As diferenças entre os tratamentos estão indicadas pelas letras que seguem as médias de larvas vivas

\begin{tabular}{lcccc}
\multicolumn{5}{c}{ larvas vivas } \\
Tratamentos & $\begin{array}{c}\text { Concentrações } \\
(\mu \mathrm{g} / \mathrm{ml})\end{array}$ & $\begin{array}{c}\text { Larvas vivas } \\
(\text { médias })^{*}\end{array}$ & $24 \mathrm{~h}(\%)$ & $48 \mathrm{~h}(\%)$ \\
Testemunha & - & $10,0 \mathrm{a}$ & 0 & 0 \\
Ilex paraguariensis & 100 & $10,0 \mathrm{a}$ & 0 & 0 \\
Melia azedarach F & 100 & $8,7 \mathrm{ab}$ & 0 & 12,9 \\
Ilex paraguariensis & 250 & $6,3 \mathrm{~cd}$ & 33,3 & 37,1 \\
Melia azedarach FL & 100 & $5,7 \mathrm{~cd}$ & 30 & 42,8 \\
Melia azedarach F & 250 & $5,3 \mathrm{e}$ & 13,3 & 47,1 \\
Ilex paraguariensis & 1000 & $3,1 \mathrm{f}$ & 56,6 & 69,3 \\
Ilex paraguariensis & 500 & $1,9 \mathrm{~g}$ & 76,6 & 80,6 \\
Melia azedarach FL & 250 & $1,8 \mathrm{~g}$ & 50 & 81,8 \\
Melia azedarach FL & 1000 & $0,5 \mathrm{~h}$ & 50 & 94,9 \\
Melia azedarach FL & 500 & $0,2 \mathrm{i}$ & 93,3 & 97,4 \\
Ilex paraguariensis & 2000 & $0,0 \mathrm{j}$ & 100 & 100 \\
Melia azedarach F & 500 & $0,0 \mathrm{j}$ & 20 & 100 \\
Melia azedarach FL & 2000 & $0,0 \mathrm{j}$ & 86,6 & 100 \\
Médias de larvas vivas apos 48 horas, seguidas por Letras que diferem entre si, pelo teste de Duncan ao nivelde 5\% de probabilidade de erro.
\end{tabular}




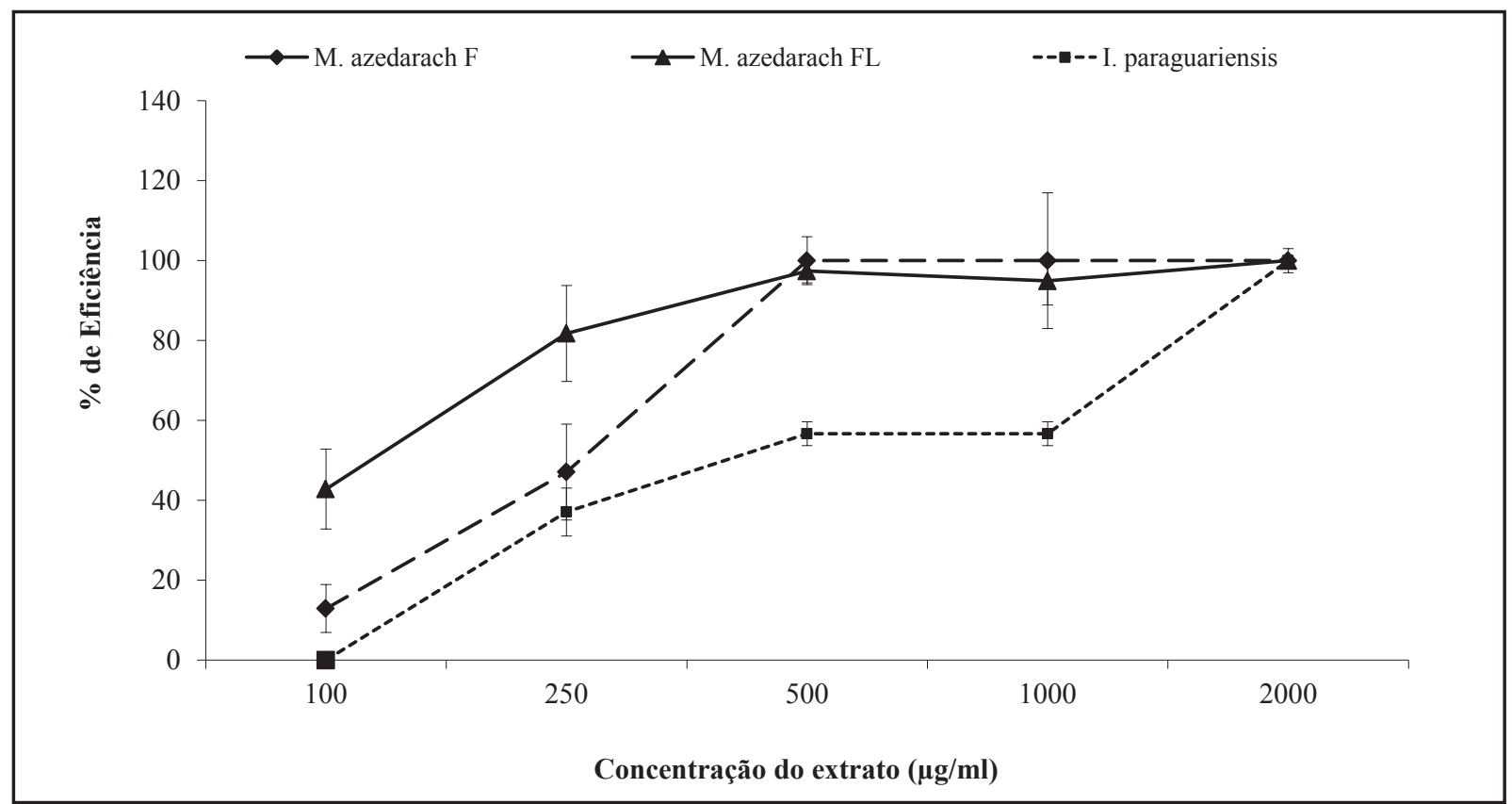

Figura 1: Eficiência dos extratos de $M$. azedarach e I. paraguariensis sobre larvas de A. aegypti em relação às diferentes concentrações, após $48 \mathrm{~h}$ de exposição.

\section{Discussão}

Os resultados obtidos demonstram que os extratos etanólicos de $M$. azedarach apresentaram atividade larvicida em grande parte das concentrações avaliadas (Tabela 1). A utilização de extratos das folhas e dos frutos da $M$. azedarach sobre $A$. aegypti com atividade, tanto larvicida quanto ovicida, já foi demonstrado em estudos anteriores (CORIA et al., 2007; ROSSI et al., 2007; VALLADARES et al., 1997), indicando que os extratos etanólicos de folhas e frutos dessa planta apresentam potencial larvicida sobre esses insetos.

O extrato de $M$. azedarach foi o mais eficiente dentre os tratamentos. Em 48 h, este apresentou 100\% de eficiência, quando utilizado a uma concentração de $500 \mu \mathrm{g} / \mathrm{ml}$ e $2000 \mu \mathrm{g} / \mathrm{ml}$, para folhas e frutos, respectivamente. Esses dados apontam que os extratos de diferentes partes da planta podem apresentar atividade larvicida.

Extratos de $M$. azedarach, além de atuação larvicida sobre A. aegypti, apresentaram eficácia sobre larvas de outros insetos, como apresentado por Migliorini et al. (2010), que testaram extrato de folhas frescas de $M$. azedarach em Diabrotica speciosa, obtendo resultado satisfatório. Cabral et al. (2008) também testaram extrato de sementes de $M$. azedarach sobre Musca domestica e obtiveram redução da fase larval em todas as amostras do extrato.

É relatado que os princípios ativos presentes em $M$. azedarach estão distribuídos nas suas diversas estruturas, embora em concentrações variáveis (BRUNHEROTTO; VENDRAMIN, 2001). Foi verificado neste estudo que a concentração de extrato dos frutos de $500 \mu \mathrm{g} / \mathrm{ml}$ foi totalmente eficiente e, nas concentrações, menores houve redução gradativa do efeito sobre as larvas, tanto em $24 \mathrm{~h}$ como em $48 \mathrm{~h}$.

Em estudo realizado por Scapinello et al. (2014), foi testado extrato do fruto de $M$. azedarach obtido através do solvente dióxido de carbono supercrítico sobre o desenvolvimento de Spodoptera frugiperda (SMITH, 1797) do período larval até a fase adulta, onde observou-se que a viabilidade das larvas de $S$. frugiperda foi significativamente alterada pela presença do extrato, com uma redução da viabilidade proporcional ao aumento da concentração de extrato adicionado à dieta. A ação larvicida da $M$. azedarach está associada à presença de compostos da classe dos terpenos, como, por exemplo, a melianona e a azadiractina, que têm comprovada ação sobre larvas e insetos (FREITAS, 2008; ROSSI et al., 2007).

Neste estudo, o bioensaio utilizando folhas de I. paraguariensis apresentou eficiência larvicida sobre A. aegypti, com $100 \%$ de mortalidade na concentração de 2000 g/ ml. Entretanto, constatou-se ação larvicida a partir da concentração de $250 \mu \mathrm{g} / \mathrm{ml}$. Destaca-se a eficiência de letalidade da $I$. paraguariensis, em que a concentração de $500 \mu \mathrm{g} / \mathrm{ml}$ apresentou superior a $80 \%$. De origem sul americana, a erva-mate ocorre naturalmente na Argentina, no Brasil e no Paraguai. No Brasil, distribui-se entre os Estados do Mato Grosso do Sul, São Paulo, Paraná, Santa Catarina e Rio Grande do Sul. A região Sul é a maior produtora, com uma produção anual aproximada de 650.000 toneladas de folhas (MACCARI e SANTOS, 2000). As folhas são moídas e utilizadas pela população na forma de chá, chimarrão e/ou tererê. Após o uso, sobra um excedente de folhas moídas que, normalmente, são descartadas como lixo orgânico. Trata-se de um volume significativo e de geração contínua que pode representar uma 
alternativa importante no controle populacional do mosquito A. aegypti.

Ilex paraguariensis é uma espécie vegetal que tem importância social e econômica, especialmente nas regiões sul do Brasil, na Argentina, no Paraguai e no Uruguai, devido ao hábito do consumo do chimarrão e do tererê (GIBERTI, 1995; SCHUBERT et al., 2006), cujo consumo pode trazer benefícios para à saúde, pois as folhas possuem substâncias antioxidantes (KOWALCZYK, 2004; CHAICOUSKI et al., 2014) e substâncias de interesse para a medicina, usadas como diuréticos (MAZZAFERA, 1994). A ação larvicida de I. paraguariensis pode estar associada à presença de metabólitos secundários da classe dos alcaloides presentes nas folhas da planta, tais como cafeína, teofilina e teobromina (BELTRAME et al., 2009).

\section{Conclusão}

Os extratos de frutos e folhas de $M$. azedarach apresentaram efeito larvicida sobre $A$. aegypti em condições de laboratório. Nas mesmas condições, os extratos de folhas de I. paraguariensis também apresentaram ação larvicida. Estes resultados abrem a perspectiva da substituição de pesticidas sintéticos por produtos naturais nos programas de controle vetorial da dengue e, mais recentemente, do Chikungunya. A eficiência dos extratos de I. paraguariensis e $M$. azedarach são promissores. A possibilidade do uso dos extratos destas plantas como larvicida para A. aegypti representa uma alternativa abundante e acessível na região sul do Brasil, onde tem sido verificado um crescimento da infestação e dos casos de dengue na última década. Além disso, surge, também, como uma opção frente ao produto sintético recomendado pelo Ministério da Saúde para o controle da dengue. Melhores resultados poderão ser obtidos com o desenvolvimento de estudos adicionais, avaliando a atividade larvicida de compostos puros isolados destas plantas.

\section{Agradecimento}

Pesquisa com apoio (bolsa) do PIBITI/CNPq e da Universidade Comunitária da Região de Chapecó UNOCHAPECÓ.

\section{Referências}

ABBOTT, W. S. A Method of Computing the Effectiveness of an Insecticide. Journal of Economic Entomology, v. 18, p. 265-267, 1925.

AUGUSTO, L. G. S.; CÂMARA NETO H. F. O combate químico das pragas domésticas: um problema oculto de saúde pública. In: Augusto LGS, Carneiro RM,
Martins PH. Abordagem ecossistêmica em saúde ensaios para o controle da dengue. Recife, Universitária UFPE, 2005, p.199-212.

BARRETO, C. F. Aedes aegypti - Resistência aos inseticidas químicos e as novas alternativas de controle. Revista Eletrônica Faculdade Montes Belos, Goiás, v.1, n.2, p.62-73, nov. 2005.

BELTRAME, J. M.; BRITO, T. O.; COTTICA, S. M. Caracterização química e físico-química do produto erva-mate: teores de umidade, cinzas, cafeína, glicose e sacarose. Anais do I Seminário Internacional de Ciência, Tecnologia e Ambiente, UNIOESTE, Cascavel, PR, 2009.

BRUNHEROTTO, R. VENDRAMIM, J. D. Bioatividade de Extratos Aquosos de Melia azedarach L. Sobre o Desenvolvimento de Tuta absoluta (Meyrick) (Lepidoptera: Gelechiidae) em Tomateiro. Neotropical Entomology, v. 30, n. 3, p. 455-459, 2001.

CABRAL, M. M. O. et al. Antimoulting activity in Brazilian Melia azedarach. Memórias do Instituto Oswaldo Cruz, v. 91, n. 1, p.117-118, 1996.

CABRAL, M. M. O et al. Melia azedarach L. extracts and their activity on Musca domestica L. (Diptera: Muscidae). Brazilian Journal of Pharmacognosy, v. 18 (Sup.), p. 699-702, 2008.

CHAICOUSKI, A; SILVA, J. E. da; TRINDADE, J. L. F. da; CANTERI, M. H. G. Determinação da quantidade de compostos fenólicos totais presentes em extratos líquido e seco de erva-mate (Ilex paraguariensis). Revista Brasileira de Produtos Agroindustriais, Campina Grande, v.16, n. 1, p. 33-41, 2014.

CORIA, C. et al. Larvicide and oviposition deterrent effects of fruit and leaf extracts from Melia azedarach L. on Aedes aegypti (L.) (Diptera: Culicidae). Bioresource Technology, v. 99, p. 3066-3070, 2008.

DONALÍSIO, M. R.; GLASSER, C. M. Vigilância entomológica e controle de vetores do dengue. Revista Brasileira de Epidemiologia, v. 5, n. 3, p. 259-271, 2002.

FREITAS, S. R. Q. Bioatividade de extratos aquosos de Eucalyptus sp. L'Hér. (Myrtaceae) e Melia azedarach L. (Meliaceae) sobre Musca domestica L. (Diptera, Muscidae). 2008. 80f. Dissertação de Mestrado, Universidade Federal de Pelotas, Brasil, 2008.

GILBERTI, G. C. Em Erva mate - Biologia e Cultura no Cone Sul. WINGE, H., FERREIRA, A. G.; MARIATH, J. E. A.; TARASCONI, L. C., Orgs.; Ed. da Universidade Federal do Rio Grande do Sul: Porto Alegre,1995, 289 p. 
KOWALCZYK, S. A Utilização do Mate (Ilex paraguariensis St.-Hill) - Um Antioxidante Natural como Estratégia para a Promoção da Saúde: Um Estudo Experimental. 2004. 90f. Tese (Enfermagem) Fundação Universidade Federal do Rio Grande Rio Grande, Rio Grande, RS, 2004.

KRINSKI, D.; MASSAROLI, A.; MACHADO, M. Potencial inseticida de plantas da Família Annonaceae. Rev. Bras. Frutic, v. 36, edição esp., e., p. 225-242, fev., 2014

LASNEAUX, Marcello Vieira. O uso de inseticidas na saúde pública: uma crítica ao modelo de combate à dengue no Brasil e no DF. 2013. 113 f. Dissertação (Mestrado em Bioética) - Universidade de Brasília, Brasília, 2013.

MACCARI, A. J.; SANTOS, A. P. R. Produtos alternativos e desenvolvimento da tecnologia industrial na cadeia produtiva da erva-mate. MCT/CNPq/PADCT, Curitiba, PR, 2000.

MAZZAFERA, P. Caffeine, theobromine and theophylline distribution in Ilex paraguariensis. Revista Brasileira de Fisiologia Vegetal, v. 6, n. 2, p. 149-151, 1994.

MIGLIORINI, P.; LUTINSKI, J. A.; GARCIA, F. R. M. Eficiência de extratos vegetais no controle de Diabrotica speciosa (Germar, 1824) (Coleoptera: Chrysomelidae), em laboratório. Biotemas, v. 23, n. 1, p. 83-89, 2010.

NEVES, D. P. Parasitologia Humana. 11. ed., Editora Abreu. São Paulo, 2005, p. 355- 367.

POHLIT, A. M.; et al. Screening of plants found in the State of Amazonas, Brazil for larvicidal activity against Aedes aegypti larvae. Acta Amazônica, v. 34, n. 1, p. 97-105, 2004.

ROSSI, J. C. N. et al. Efeito Larvicida de Extratos Etanólicos de Folhas Secas e Frutos Maduros de Melia azedarach (Meliaceae) sobre Aedes albopictus. Latin American Journal of Pharmacy, v. 26, n. 5, p. 737-40, 2007.

SANTA CATARINA. DIVE. Diretoria de Vigilância Epidemiológica. Secretaria de Estado da Saúde: Superintendência de Vigilância em Saúde. Informações Dengue. 2014. Florianópolis. Disponível em: $<\underline{\mathrm{http}}$ :// www.dive.sc.gov.br/index.php?option $=\mathrm{com}$ content\&task=view\&id=140\&Itemid=151> Acesso em: 10 abr. 2014.

SCAPINELLO, J. et al. Insecticidal and growth inhibiting action of the supercritical extractsof Melia azedarach on Spodoptera frugiperda. Revista Brasileira de Engenharia Agrícola e Ambiental, v. 18, n. 8, p. 866-872, 2014.
SCHUBERT, A.; ZANIN, F. F.; PEREIRA, D. F.; ATHAYDE, M. L. Variação anual de metilxantinas totais em amostras de Ilex paraguariensis A. ST. - HIL. (erva mate) em Ijuí e Santa Maria, Estado do Rio Grande do Sul. Quim. Nova, v. 29, n. 6, p. 1233-1236, 2006.

TAUIL, P. L. Aspectos críticos do controle do dengue no Brasil. Cad. Saúde Pública, v. 18, n. 3, p. 867-871, 2002.

VALLADARES, G.; DEFAGO, M. T.; PALACIOS, S. Laboratory evaluation of Melia azedarach (Meliaceae) extracts against the Elm Leaf Beetle (Coleoptera: Chrysomelidae). Journal of Economic Entomology, v. 90, n. 3, p. 747-750, 1997. 